\title{
Efeito de vídeo educativo no comportamento de higiene bucal de pacientes hematológicos*
}

\author{
Effects of an educational video on the oral hygiene of patients with hematologic disorders
}

Efecto de video educativo en el comportamiento de higiene bucal de pacientes hematológicos

Emilia Campos de Carvalho ${ }^{1}$, Ana Paula Neroni Stina ${ }^{2}$, Milene Thaís Marmol ${ }^{3}$, Livia Maria Garbin ${ }^{4}$, Fernanda Titareli Merizio Martins Braga ${ }^{5}$, Lucimara Moreli ${ }^{6}$, Cristina Mara Zamarioli ${ }^{7}$

\footnotetext{
* Projeto foi desenvolvido com financiamento do Conselho Nacional de Desenvolvimento Científico e Tecnológico (CNPq).

${ }^{1}$ Enfermeira, Doutora em Enfermagem. Professora Titular da Escola de Enfermagem de Ribeirão Preto da Universidade de São Paulo (EERP/USP). Bolsista

Produtividade do CNPq. Ribeirão Preto, SP, Brasil. E-mail: ecdcava@eerp.usp.br.

2 Discente do curso de graduação em Enfermagem da EERP/USP. Bolsista de Iniciação Científica do CNPq. Ribeirão Preto, SP, Brasil. E-mail: aninha_stina@yahoo.com.br.

${ }^{3}$ Enfermeira. Enfermeira do Centro Infantil Boldrini. Campinas, SP, Brasil. E-mail: milene.marmol@usp.br.

${ }^{4}$ Enfermeira, Doutora em Ciências. Enfermeira da EERP/USP. Ribeirão Preto, SP, Brasil. E-mail: liviagarbin@usp.br.

${ }^{5}$ Enfermeira, Doutora em Ciências. Enfermeira Especialista em Laboratório da EERP/USP. Ribeirão Preto, SP, Brasil. E-mail: titareli@eerp.usp.br.

${ }^{6}$ Enfermeira, Mestre em Ciências. Enfermeira da Fundação Hemocentro de Ribeirão Preto. Ribeirão Preto, SP, Brasil. E-mail: lucimara.moreli@usp.br.

${ }^{7}$ Enfermeira. Discente do Programa de Pós-Graduação em Enfermagem Fundamental, nível Mestrado, da EERP/USP. Ribeirão Preto, SP, Brasil. E-mail: crisitna_zamarioli@yahoo.com.br.
}

\section{RESUMO}

Este estudo teve como objetivo avaliar a eficácia e a aceitação de um vídeo como estratégia de ensino da higiene bucal para pacientes com doenças hematológicas em tratamento quimioterápico. Trata-se de estudo quase experimental, tipo antes-depois, no qual foram incluídos 23 sujeitos. Depois de observada a técnica de higiene bucal habitual, o paciente assistiu a um vídeo sobre este procedimento. Posteriormente, o sujeito realizou novamente a higienização bucal. Aplicou-se o teste t de "Student" pareado para comparar as médias de acertos dos passos desta técnica antes e após apresentação do vídeo. O desempenho na realização do procedimento foi superior após os sujeitos assistirem ao vídeo ( $p<0.0001)$, sendo que a utilização desta estratégia foi bem-aceita pelos pacientes. $O$ vídeo sobre a técnica de higiene bucal mostrou-se eficaz para o ensino deste procedimento e foi bem aceito pelos pacientes. (ClinicalTrials.gov Identifier: NCT 01718002)

Descritores: Cuidados de Enfermagem; Educação; Higiene bucal; Quimioterapia.

\section{ABSTRACT}

The current study aims at evaluating the effectiveness and acceptance of a video as a strategy for teaching oral hygiene to patients with hematologic disorders receiving chemotherapy. It is a quasi-experimental before and after study, composed of 23 subjects. After their routine oral hygiene routine was observed, patients watched a video about the procedure. Afterwards, the subjects carried out their oral hygiene once more. A paired student's t-test was used to compare the average of how many steps were performed correctly before and after the video presentation. Procedure performance was higher after watching the video $(p<0.0001)$, being that the strategy was well accepted by patients. The oral hygiene video proved to be effective for the teaching of this procedure and was well accepted by patients.

(ClinicalTrials.gov Identifier: NCT 01718002)

Descriptors: Nursing Care; Education; Oral Hygiene; Drug Therapy.

\section{RESUMEN}

Estudio que objetivó evaluar la eficacia y aceptación de un video como estrategia de enseñanza de la higiene bucal en pacientes con enfermedades hematológicas en tratamiento quimioterápico. Estudio cuasi-experimental, tipo antesdespués, en el que fueron incluidos 23 sujetos. Luego de observada la técnica de higiene bucal habitual, el paciente vio un video sobre dicho procedimiento. Posteriormente, el sujeto realizó nuevamente la higienización bucal. Se aplicó test $t$ de Student pareado, para comparar los promedios de acierto de los pasos de esta técnica antes y después de la presentación del video. El desempeño en la realización del procedimiento fue superior luego de ver el video $(p<0,0001)$, siendo que la utilización de esta estrategia fue bien aceptada por los pacientes. El video sobre la técnica de higiene bucal se mostró efectivo para la enseñanza del procedimiento y fue bien aceptado por los pacientes. (ClinicalTrials.gov Identifier: NCT 01718002)

Descriptores: Atención de Enfermería; Educación; Higiene Bucal; Quimioterapia. 


\section{INTRODUÇÃO}

A educação em saúde é atividade inerente ao processo de trabalho do enfermeiro, sendo que tradicionalmente utiliza-se a orientação verbal enquanto estratégia de ensino-aprendizagem.

No entanto, conforme apontado na literatura, a orientação verbal pode não ser efetiva, podendo ser prejudicada por diversos aspectos como a linguagem utilizada pelo profissional e a falta de concentração dos pacientes $^{(1)}$. Estes mesmos autores citam que o profissional pode lançar mão de alternativas para facilitar este processo, tais como a orientação de pacientes em grupo, realização de oficinas, utilização de vídeos e painéis com fotos, dentre outros, os quais agradam os pacientes e contribuem para o seu aprendizado.

Nesse sentido, a presença das tecnologias no cotidiano da enfermagem, em especial no ambiente de trabalho e de cuidado, tem se tornado crescente e indispensável atualmente; sendo que quando as mesmas são utilizadas e administradas de forma adequada e inteligente, podem beneficiar a prática do cuidado ao ser humano em múltiplas esferas ${ }^{(2)}$. Uma possibilidade de emprego de tecnologias é a utilização de vídeos ${ }^{(3)}$.

O vídeo consiste em uma estratégia audiovisual de maior impacto na aprendizagem quando comparada com a linguagem escrita, pois possui uma série de imagens com movimentos gráficos, textos e sons que permitem ao espectador melhor compreensão do tema apresentado, facilitando a construção do conhecimento, principalmente se possui até 10 minutos de duração ${ }^{(3-4)}$. Diversas experiências foram descritas com sucesso empregando o vídeo no ensino de pacientes ou população que exige conhecimento para o autocuidado ou cuidado de familiares ${ }^{(5-6)}$.

Frente às vantagens da utilização do vídeo apresentadas na literatura, esta pode ser uma estratégia utilizada para a educação dos pacientes em relação à higiene bucal.

Tem sido evidenciada a importância dos pacientes receberem um melhor preparo educacional relacionado à higienização da boca, sendo essa relevância intensificada quando se aplica a pessoas em tratamento quimioterápico. Dentre as toxicidades relacionadas ao tratamento do câncer que acometem a cavidade bucal são citadas a alteração do paladar, candidíase, disfagia, gengivite, infecção, mucosite, periodontite, ulceração e xerostomia $^{(7-8)}$.

Além das alterações locais, é necessário considerar que a cavidade bucal pode ser o foco de origem para a disseminação de organismos patogênicos para locais distantes do corpo, especialmente em sujeitos imunocomprometidos, de forma que a leucopenia associada ao tratamento do câncer pode aumentar a suscetibilidade à ocorrência de bacteremia de origem oral, eventualmente causando sepse e até mesmo a morte $e^{(7)}$.

Em revisão sistemática da literatura que teve como objetivo identificar as complicações bucais relacionadas à quimioterapia em crianças portadoras de leucemia linfoide aguda, é ressaltado que a prevalência destas manifestações foi relacionada não apenas ao tratamento quimioterápico, mas também à má higienização bucal dos pacientes ${ }^{(8)}$.

Assim, é cada vez mais reconhecido que a higiene bucal é parte essencial dos cuidados aos pacientes com câncer, pois as complicações orais têm impacto na qualidade de vida, nos custos relacionados ao cuidado e no prolongamento ou comprometimento do tratamento $^{(9)}$,interferindo nos resultados da terapêutica.

A higiene bucal é composta por diversas etapas, que incluem a escovação, uso de fio ou fita dental e uso de enxaguatório bucal. A escovação é reconhecida como a medida mais eficiente e usual para remover o biofilme dental $^{(10-11)}$. Para a realização desta etapa, levando em consideração a sensibilidade do tecido bucal de pacientes com câncer, faz-se necessário o uso de técnica e instrumentos apropriados ${ }^{(12)}$.

Nesse contexto, ressalta-se que a qualidade da higiene bucal antes e durante a terapia, associada à duração do tratamento, tipo, combinação de antineoplásicos e idade, podem contribuir para a intensidade das complicações da cavidade bucal dos pacientes $^{(13)}$, necessitando os mesmos serem devidamente capacitados para a realização do procedimento sem que o mesmo ofereça mais riscos.

Desta maneira, a presente pesquisa, que teve como objetivo avaliar a eficácia e a aceitação de um vídeo como estratégia de ensino do procedimento de higienização bucal em pacientes portadores de malignidades hematológicas; tem a finalidade de favorecer o paciente no desenvolvimento de competências para executar esse procedimento, alicerçado nas premissas de que a higienização inadequada altera a saúde bucal dos pacientes em tratamento quimioterápico e pode ser melhorada em parte significativa dos pacientes.

\section{METODOLOGIA}

Pesquisa quase experimental, tipo antes-depois, sendo a intervenção empregada a utilização de um vídeo 
educativo sobre higienização bucal. Para tanto, foram avaliados os passos adotados pelos sujeitos para higiene bucal antes (desempenho inicial) e após (desempenho final) a exposição ao vídeo educativo.

O estudo foi realizado em uma unidade de internação de pacientes com doenças hematológicas de um hospital escola paulista. Os critérios adotados para inclusão dos sujeitos foram: estar internado a menos de 24 horas; condições físicas para o autocuidado durante a higiene bucal, ou seja, apresentar escore $\leq 3$ de acordo com a Escala de Desempenho do Estado Geral do Paciente, elaborada e aprovada pelo American Eastern Cooperative Oncology Group (ECOG) ${ }^{(14)}$; aceitar dieta oral; ausência de cateter nasal, máscaras de oxigenação, sondas nasogástrica ou nasoentérica; ausência de patologias, alterações ou lesões bucais que impediam a realização do procedimento; presença de dentes; e apresentar contagem de plaquetas $>20.000 / \mathrm{mm}^{3}$ e de neutrófilos $\geq 1.000 / \mathrm{mm}^{3}$.

Prezando pela segurança dos pacientes, a adoção de contagem de plaquetas $>20.000 / \mathrm{mm}^{3}$ como critério de inclusão se deve ao fato de que, quando em valores inferiores a $50.000 / \mathrm{mm}^{3}$, os pacientes apresentam risco moderado de sangramento ${ }^{(15)}$ e em valores abaixo de $20.000 / \mathrm{mm}^{3}$ deve ser considerada a possibilidade de transfusão profilática(16). Em relação à contagem de neutrófilos, a intensidade do risco de infecção em pacientes com câncer está diretamente relacionada ao grau de neutropenia, sendo que abaixo de $1000 / \mathrm{mm}^{3}$ apresentam risco moderado e abaixo de $500 / \mathrm{mm}^{3}$ risco severo de adquirir infecções ${ }^{(15)}$.

A avaliação dos pacientes e aplicação do vídeo foram feitas por três avaliadores, sendo duas enfermeiras e uma aluna do terceiro ano do curso de graduação em enfermagem. Antes do início da coleta de dados foi realizado treinamento dos avaliadores e um estudo piloto com três pacientes para avaliação dos instrumentos. Não foram necessárias alterações nos instrumentos, porém os pacientes relataram que, ao invés de assistirem ao vídeo por duas vezes seguidas, conforme havia sido proposto inicialmente, apenas uma vez seria suficiente e menos cansativo. Ressalta-se que os participantes do estudo piloto não foram incluídos na amostra do estudo.

Para a coleta dos dados foi utilizado instrumento ${ }^{(17)}$ já validado e aplicado anteriormente em uma população com características similares, composto por variáveis sociodemográficas (idade, sexo, etnia e ocupação), etilismo, tabagismo, valor atribuído à importância da higienização bucal, horários e procedimentos empregados durante a higienização bucal e a aceitação da estratégia educativa.

Para a avaliação da importância da higienização bucal e da aceitação da estratégia educativa, foi solicitado ao paciente que atribuísse um valor em uma escala de zero a 10.

O procedimento de higienização bucal foi avaliado segundo uma lista contendo 15 passos (escore de zero a $15)$, os quais correspondem ao conteúdo apresentado no vídeo desenvolvido para este estudo.

O vídeo, com aproximadamente 10 minutos, contém ilustrações, filmagem e narração dos materiais e movimentos empregados durante o procedimento de higiene bucal, e foi validado quanto à aparência e conteúdo.

O projeto foi submetido ao Comitê de Ética em Pesquisa da instituição onde o estudo foi desenvolvido e aprovado de acordo com o Processo n 5696/2010. O estudo foi desenvolvido de novembro de 2011 a junho de 2012.

Neste período foram realizadas visitas diárias à enfermaria e ocorreram 159 internações, as quais totalizaram 93 pacientes rastreados, tendo em vista que cerca de $50 \%$ deles apresentaram mais de uma internação. O paciente era abordado na primeira internação e, caso não atendesse aos critérios de elegibilidade e fosse internado novamente posteriormente, ele era novamente abordado. Nova abordagem só não era realizada se o paciente se recusasse a participar desde o primeiro momento, ou se ele apresentasse algum critério de elegibilidade não modificável, por exemplo, a ausência de dentes e uso de prótese total.

Assim, dos 93 pacientes rastreados, 24 atenderam aos critérios de elegibilidade. Dos 69 restantes, 14 recusaram-se a participar e 55 não preencheram os critérios de inclusão (27 por plaquetopenia e/ou neutropenia; dois por apresentarem lesões na boca e/ou sangramento; três por uso de sondas e/ou máscaras de oxigenação; 11 por ausência total de dentes e 12 por motivos relacionados a curto tempo de internação no setor, liberação dos resultados dos exames laboratoriais fora do prazo estabelecido para coleta de dados ou realização de procedimento diagnóstico incompatível com a coleta). Um dos sujeitos solicitou interrupção de sua participação no estudo, assim, a amostra foi composta por 23 sujeitos.

Nas primeiras 24 horas de internação, o paciente elegível era informado quanto às características e objetivos do estudo e convidado a participar. Obtido o 
consentimento verbal, era solicitada a assinatura do termo de consentimento livre e esclarecido, em duas vias, sendo uma entregue ao sujeito e outra arquivada juntamente com a documentação essencial, sob a posse do investigador principal.

Após aplicação do instrumento para caracterização do sujeito, foi fornecido material para higiene bucal individual (estojo contendo escova de cerdas macia, dentifrício com baixo teor de abrasividade, fio dental e enxaguatório bucal, cujo uso seguiu a prescrição médica, sendo variável entre os pacientes). O sujeito foi orientado a executar a técnica de higienização bucal habitual, sendo os materiais, a sequência e os movimentos empregados avaliados e registrados no instrumento para análise dos passos. Em seguida, o paciente assistiu ao vídeo, na própria enfermaria em que estavam internados, em um notebook protegido por uma proteção plástica que era removida e descartada na própria enfermaria após o uso, respeitando as regras de proteção do ambiente e do paciente. Após realizou novamente o procedimento, enquanto o avaliador observava e registrava no instrumento os materiais, a sequência e os movimentos empregados.

Por fim, foi solicitado que o paciente atribuísse um valor, numa escala de zero a dez, para a aceitação do vídeo como estratégia de aprendizado da higienização bucal.

Os dados foram inseridos em planilha eletrônica do aplicativo Excel $^{\circledR}$ e analisados no programa Statistical Package for Social Science (SPSS), versão 17.0 for Windows.

As variáveis de caracterização clínica e demográfica foram apresentadas utilizando a frequência absoluta e porcentagem. Depois de verificada a distribuição normal, pelo teste de Shapiro Wilk, das médias de acertos obtidos pelos sujeitos na realização do procedimento, foi utilizado o teste t "Student" pareado para comparar a diferença entre as médias de acertos obtidos pelos sujeitos antes e após apresentação do vídeo.

\section{RESULTADOS}

A amostra $(n=23)$ foi composta por seis mulheres $(26,1 \%)$ e 17 (73,9\%) homens; com idade média de 42,9 anos (mínimo de 18 e máximo de 78 anos).

Quando questionados sobre a importância da higiene bucal, os pacientes atribuíram um valor médio de 9,5 (desvio padrão=4,39, variação de oito a 10).

Em relação à realização da higiene bucal ao longo do dia, os mesmos referiram uma frequência que variou de duas a quatro vezes, sendo em média 3,3 vezes ao dia.
Sobre o momento do dia no qual a higiene bucal habitualmente é realizada, a predominância é ao acordar $(n=21 ; 91,3 \%)$, após o almoço $(n=20 ; 86,9 \%)$ e antes de dormir $(n=19 ; 82,6 \%)$. Outros momentos citados foram após o café da manhã $(n=3 ; 13,0 \%)$, após o lanche $(n=5 ; 21,7 \%)$ e após o jantar $(n=7 ; 30,4 \%)$.

Inicialmente foi solicitado aos sujeitos que demonstrassem a higiene bucal, conforme a realizam habitualmente, antes de assistirem ao vídeo. Dentre os 15 passos avaliados na execução do procedimento, a média de acertos foi de 6,8 (45,3\%, variação de zero a 12 passos). Depois de assistirem ao vídeo, houve um aumento para 11,6 passos realizados corretamente (77,3\%, variação de sete a 15 passos) (Figura 1 ). Essa diferença representou um aumento médio de 32 pontos percentuais, sendo a mesma estatisticamente significante $\left(t_{22 ; 0,05}=-6,567 ; p<0,0001\right)$. 


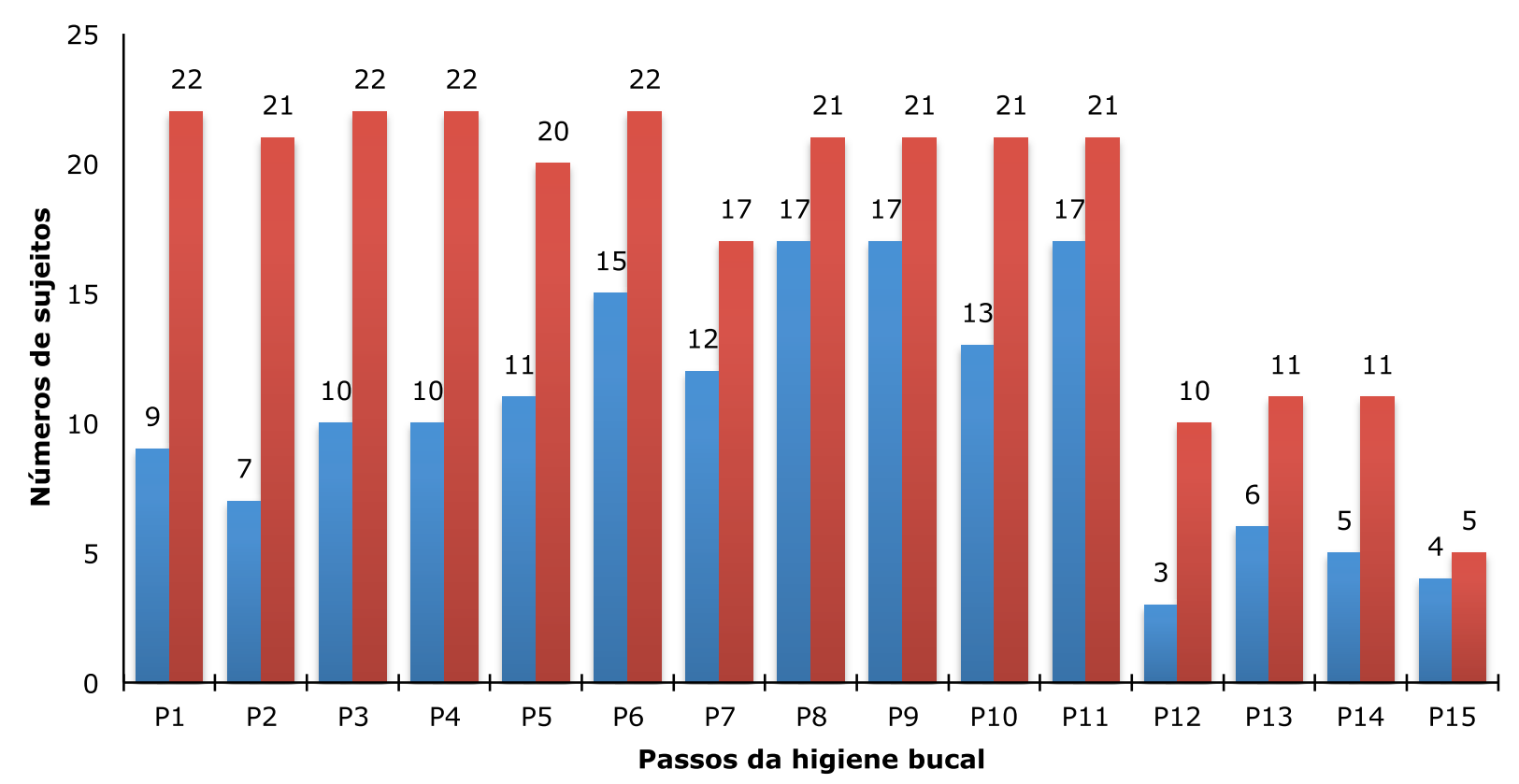

P1 (passo 1) - Aplicou uma pequena quantidade de creme dental na escova;

P2 - Colocou as cerdas da escova inclinadas no local adequado, entre a gengiva e o dente; Colocou as cerdas da escova inclinadas no local adequado, entre a gengiva e o dente;

P3 - Escovou a arcada superior com movimentos de varredura no sentido de cima para baixo;

P4 - Escovou a arcada inferior com movimentos de varredura de cima para baixo;

P5 - Escovou a parte interna dos dentes da gengiva em direção a ponta do dente, empregando o movimento de "varrer";

P6 - Posicionou a escova sobre a superfície mastigatório dos dentes na posição horizontal, realizando movimentos suaves de vai e vem em todos os dentes;

P7 - Repetiu cada movimento de 5 a 10 vezes;

P8 - Cuspiu a espuma formada sempre que necessário;

P9 - Enxaguou a escova e a boca;

P10 - Escovou a língua do fundo para a frente, com as cerdas da escova, como se estivesse varrendo-a, até remover a placa formada na língua;

P11 - Enxaguou a boca novamente;

$\mathbf{P 1 2}$ - Com cerca de $30 \mathrm{~cm}$ de fio/fita dental, prendeu a maior parte no dedo médio de uma das mãos e o restante no dedo médio da outra;

P13 - Prendeu o fio/fita esticado entre o polegar e o indicador, mantendo um pequeno espaço entre eles;

P14 - Deslizou suavemente o fio/fita entre os dentes, curvando-o formando um "C" sobre a superfície de cada dente e deslizando entre o dente e a gengiva;

P15 - Utilizou cerca de $10 \mathrm{ml}$ de enxaguatório bucal, bochechando por 1 minuto.

Figura 1: Distribuição dos sujeitos segundo passos da higiene bucal executados adequadamente, antes e após o vídeo educativo. Ribeirão Preto/SP, 2012.

O desempenho dos sujeitos, segundo o passo observado, apresentou ampla variação. Ainda, na Figura 1 é possível observar cada um dos passos avaliados e a média de acertos antes e após assistirem ao vídeo. Os passos realizados corretamente pelo maior número de sujeitos antes de assistirem ao vídeo foram o oito, nove e 11 ( $73 \%$ cada), os quais estão relacionados ao ato de cuspir a espuma formada, enxaguar a boca e a escova. Em contrapartida, os que apresentarem menor frequência de acertos foram os passos $12,13,14$ e 15 $(13,26,22$ e $17 \%$ respectivamente), os quais tratam da utilização do fio/fita dental, bem como do enxaguatório bucal.
Ao realizarem o procedimento novamente após assistirem ao vídeo, os passos de um a 11, que dizem respeito à escovação propriamente dita, apresentaram considerável aumento no número de acertos, sendo que, dentre esses, o que teve o menor incremento foi o passo 7, ou seja, a repetição de cada movimento da escovação de cinco a 10 vezes. Contudo, ao avaliar os passos relacionados ao uso de fio/fita dental, e também do enxaguatório bucal, menos da metade dos sujeitos os realizou corretamente.

Enquanto a maior diferença entre os dois momentos foi no passo dois (inclinação adequada da escova), que foi realizado corretamente por $30 \%$ dos sujeitos antes e 
por 91\% após a intervenção; a menor diferença foi no passo 15 (uso do enxaguatório bucal), cujo índice de acertos se mostrou baixo em ambos os momentos.

Quando questionados acerca da aceitação do vídeo como estratégia de aprendizagem, os sujeitos atribuíram notas que variaram de oito e 10 (média de 9,8 e desviopadrão de 2,3).

\section{DISCUSSÃO}

É possível que a amostra deste estudo represente o padrão de comportamento de adultos relacionado à higiene bucal, como já descrito na literatura, na qual é mostrado que mesmo possuindo um nível de conhecimento razoável, os sujeitos não a realizam de maneira adequada(18). Este fato passa a ter maior relevância ao se considerar os riscos de infecção que os sujeitos do estudo estão expostos, em especial pela imunossupressão decorrente da patologia de base e do tratamento.

Apesar dos pacientes terem atribuído elevada importância à higiene bucal, tanto a frequência quanto a técnica realizadas cotidianamente não se mostraram adequadas. Os resultados apresentados neste estudo quanto à falha na execução da técnica e também à frequência de realização da higiene bucal corroboram com os encontrados por outros autores, que avaliaram o hábito de higiene bucal em 478 adolescentes e adultos, e identificaram que a maioria realiza a escovação três vezes ao $\operatorname{dia}^{(19)}$

Além disso, estudo realizado com pacientes adultos de uma unidade de oncohematologia, em fase de avaliação para o início do primeiro ciclo de quimioterapia, embora não tenha avaliado aspectos relacionados à frequência e adequação da técnica, mostrou que 30\% dos sujeitos apresentavam condições de higiene bucal que variaram de deficiente a péssima(17).

Ao se considerar os passos do procedimento menos empregados (passos 12 ao 15), destaca-se a possível influência do receio de sangramento gengival como impedimento quanto à utilização do fio dental, usualmente referido por pacientes em tratamento quimioterápico. Apesar desta ser uma etapa preconizada na realização da higiene bucal, as equipes que acompanham pacientes oncohematológicos podem apresentar recomendações específicas considerando as contagens de plaquetas $^{(20)}$ e de neutrófilos. Nesse sentido destaca-se que no presente estudo a amostra foi composta por sujeitos que apresentavam contagem de plaquetas acima de $20.000 / \mathrm{mm}^{3}$ e neutrófilos iguais ou acima $1.000 / \mathrm{mm}^{3}$, situação que não impede a utilização do fio/fita dental.

Em relação ao uso de enxaguatório bucal, a falta de costume pode ter sido um dos motivos para a não incorporação deste passo no procedimento. No entanto, é necessário levar em consideração os efeitos benéficos relacionados ao uso do enxaguatório bucal. Conforme apontado em revisão sistemática da literatura que incluiu estudos realizados com pacientes adultos, com gengivite e boas condições de saúde; há fortes evidências de que os bochechos com clorexidina tem importante ação contra a formação de placas e também contra os efeitos da gengivite quando utilizado como um adjuvante da higiene bucal regularmente; sendo que quando comparado aos controles utilizados nos estudos experimentais, resultou em aproximadamente menos $33 \%$ formação de placas e $26 \%$ menos gengivite ${ }^{(21)}$.

Assim, faz-se necessário reforçar a importância do emprego do fio/fita dental e do enxaguatório bucal como coadjuvantes na higiene bucal adequada, tanto para a população em geral quanto para a clientela estudada.

Em relação à escovação dos dentes, é necessário considerar que esta é apontada como a medida mais eficiente e usual para remoção de biofilme dental ${ }^{(10-11,22)}$. Embora os escores de acerto dos passos referentes à escovação propriamente dita tenham se apresentado baixos antes dos sujeitos serem expostos ao vídeo, depois de assistirem ao mesmo os movimentos empregados e posicionamento da escova nas arcadas melhoraram em relação ao desempenho inicial, com aumento significativo dos escores; sendo que esse aumento pode retratar a efetividade da utilização do vídeo enquanto estratégia de conscientização e de ensino da população em estudo.

Nesse contexto cabe ainda destacar que o controle diário do biofilme, por meio do emprego da escovação(23), é de grande importância para a manutenção das condições de saúde bucal, evitando ou retardando a colonização do meio gengival por espécies bacterianas $^{(24)}$.

Em relação à adoção do vídeo como estratégia de ensino-aprendizagem, a literatura aponta que, para que o processo de assimilação do conteúdo seja atingido, a transmissão do conhecimento deve ser realizada de forma a favorecer a compreensão da informação transmitida ${ }^{(3)}$. Neste contexto, embora o incremento dos escores não tenha ocorrido de maneira uniforme entre os passos avaliados, observou-se melhora no número de acertos como um todo, evidenciando-se assim sua 
utilidade enquanto instrumento educacional voltado a pacientes para a aquisição de conhecimentos.

Este dado vai ao encontro de outros resultados apontados na literatura. Como exemplo, em estudo realizado com pacientes portadores de déficit visual, verificou-se que o uso dessa intervenção contribuiu para o aumentou significativo do conhecimento dos pacientes acerca do déficit visual $(p<0,001)$, além de ser uma estratégia não onerosa, quando comparada a outras tecnologias de ensino(25).

Deve-se levar em consideração que a educação e motivação promovem o aumento no nível de conhecimento dos pacientes e estão intimamente relacionados à mudança de comportamento de higiene bucal dos mesmos ${ }^{(18)}$, sendo que a avaliação satisfatória, relacionada à experiência de aprendizagem, feita pelos sujeitos do presente estudo, reforçou o caráter positivo atribuído.

\section{CONSIDERAÇÕES FINAIS}

Os resultados apresentados evidenciam que a estratégia utilizada (vídeo educativo) contribuiu para a mudança do comportamento imediato dos sujeitos, no

\section{REFERÊNCIAS}

1. Kruse MHL, Almeida MA, Keretzky KB, Rodrigues E, Silva FP, Schenini FS, Garcia VM. Orientação pré-operatória da enfermeira: lembranças de pacientes. Rev. Eletr. Enf. [Internet]. 2009 [acesso em: 30 jun 2014];11(3):494-500. Disponível em: http://www.fen.ufg.br/revista/v11/n3/v11n3a05.htm.

2. Baggio MA, Erdmann AL, Dal Sasso GTM. Cuidado humano e tecnologia na enfermagem contemporânea e complexa. Texto Contexto Enferm [Internet]. 2010 [acesso em: 30 jun 2014];19(2):378-85. Disponível em:

http://dx.doi.org/10.1590/S0104-07072010000200021. 3. Moreira CB, Bernardo EBR, Catunda HLO, Aquino PS, Santos $\mathrm{MCL}$, Fernandes AFC. Construção de um vídeo educativo sobre detecção precoce do câncer de mama. Revista Brasileira de Cancerologia [Internet]. 2013 [acesso em: 30 jun 2014];59(3):401-7. Disponível em:

http://www.inca.gov.br/rbc/n_59/v03/pdf/10-artigo-construcaovideo-educativo-sobre-deteccao-precoce-cancer-mama.pdf. 4. Fleming SE, Reynolds J, Wallace B. Lights... camera... action! a guide for creating a DVD/video. Nurse Educ [Internet]. 2009 [acesso em: 30 jun 2014];34(3):118-21. Disponível em: http://dx.doi.org/10.1097/NNE.0b013e3181a0270e. 5. Paula AAD, Carvalho EC. Ensino sobre perioperatório a pacientes: estudo comparativo de recursos audiovisual (vídeo) e oral. Rev Lat Am Enfermagem [Internet]. 1997 [acesso em: 30 jun 2014];5(3):35-42. Disponível em: http://dx.doi.org/10.1590/S0104-11691997000300006. 6. Palmeira ILT, Silva RM, Lopes MVO, Fernandes AFC. Tecnologia audiovisual como instrumento de apoio na orientação para o auto-exame de mama. Rev. RENE [Internet]. 2004 [acesso em: 30 jun 2014];5(1):62-7. Disponível em: http://www.revistarene.ufc.br/revista/index.php/revista/article/vi ew/848.

7. Ertas ET, Kurnaz F, Zorba YO, Kocyigit I, Sisman Y, Kaynar L et al. Comparison of chemotherapy and hematopoietic stem cell transplantation pre and postterm DMFT scores: a preliminary study. Niger J Clin Pract [Internet]. 2014 [acesso em: 30 jun ambiente hospitalar, sendo esta uma mídia de fácil acesso e de retorno rápido. A estratégia foi bem aceita e representa uma importante ferramenta a ser utilizada pela equipe multidisciplinar e pode contribuir para minimizar complicações na cavidade bucal e sistêmicas, em especial em pessoas em tratamento quimioterápico.

Avaliar os passos adotados pelos sujeitos na execução técnica do procedimento possibilita a verificação de deficiências pontuais, permitindo ensino e reforço positivo quanto a melhor prática.

Ainda que os resultados na fase imediatamente posterior à exposição do vídeo sejam satisfatórios, destaca-se a contribuição de acompanhamento de sujeitos por um período de tempo maior, para verificar a mudança de comportamento, sobretudo no domicílio, pois o desempenho observado pode ser influenciado pelo reflexo da exposição imediata (memorização).

O tamanho amostral, a despeito do rastreamento intensivo, foi pequeno dadas as características dos pacientes e os rigorosos critérios de inclusão adotados (parâmetros hematológicos). Contudo, a amostra permitiu análises que evidenciaram o efeito da intervenção.

2014];17(1):32-7. Disponível em: http://dx.doi.org/10.4103/1119-3077.122831.

8. Morais EF, Lira JA, Macedo RA, Santos KS, Elias CT, Morais ML. Oral manifestations resulting from chemotherapy in children with acute lymphoblastic leukemia. Braz J Otorhinolaryngol [Internet]. 2014 [acesso em: 30 jun 2014];80(1):78-85. Disponível em: http://dx.doi.org/10.5935/1808-8694.20140015.

9. Bueno AC, Ferreira RC, Barbosa FI, Jham BC, Magalhães CS, Moreira AN. Periodontal care in patients undergoing radiotherapy for head and neck cancer. Support Care Cancer [Internet]. 2013 [acesso em: 30 jun 2014];21(4):969-75. Disponível em: http://dx.doi.org/10.1007/s00520-012-1614-5.

10. Barbosa AM, Ribeiro DM, Caldo-Teixeira AS. Conhecimentos e práticas em saúde bucal com crianças hospitalizadas com câncer. Cien Saude Colet [Internet]. 2010 [acesso em: 30 jun 2014];15 Suppl. 1:1113-22. Disponível em:

http://dx.doi.org/10.1590/S1413-81232010000700019.

11. Abidia RF. Oral care in the intensive care unit: a review. J Contemp Dent Pract. 2007;8(1):76-82.

12. Kennedy HF, Morrison D, Tomlinson D, Gibson BE, Bagg J, Gemmell CG. Gingivitis and toothbrushes: potential roles in viridans streptococcal bacteraemia. J Infect [Internet]. 2003 [acesso em: 30 jun 2014];46(1):67-70. Disponível em: http://dx.doi.org/10.1053/jinf.2002.1084.

13. Sonis S, Clark J. Prevention and management of oral mucositis induced by antineoplastic therapy. Oncology (Williston Park). 1991;5(12):11-8.

14. Oken MM, Creech RH, Tormey DC, Horton J, Davis TE, McFadden ET et al. Toxicity and response criteria of the Eastern Cooperative Oncology Group. Am J Clin Oncol. 1982;5(6):64955.

15. Bonassa EMA, Santana TR. Enfermagem em terapêutica oncológica. $3^{a}$ ed. Rio de Janeiro: Atheneu; 2005.

16. Covas DT. Suporte transfusional do paciente com neoplasia hematológica. In: Zago MA, Falcão RP, Pasquini R. Hematologia fundamentos e prática. São Paulo: Atheneu; 2004. p. 413-8. 
17. Carvalho EC, Cárnio EC, Khouri VY, Guilherme C, Santos CB, Pace MA. Exame da cavidade bucal de pacientes com câncer: avaliação clínica e dosagem indireta de óxido nítrico. Rev Esc Enferm USP [Internet]. 2013 [acesso em: 30 jun 2014];47(1):101-6. Disponível em:

http://dx.doi.org/10.1590/S0080-62342013000100013. 18. Garcia PPNS, Campos FP, Rodrigues JA, Santos PA, Dovigo LN. Avaliação dos efeitos da educação e motivação sobre o conhecimento e comportamento de higiene bucal em adultos. Cienc Odontol Bras [Internet]. 2004 [acesso em: 30 jun 2014];7(3):30-9. Disponível em:

http://ojs.fosjc.unesp.br/index.php/cob/article/view/304. 19. Lisbôa IC, Abegg C. Hábitos de higiene bucal e uso de serviços odontológicos por adolescentes e adultos do Município de Canoas, Estado do Rio Grande do Sul, Brasil. Epidemiol. Serv. Saúde [Internet]. 2006 [acesso em: 30 jun 2014];15(4):29-39. Disponível em: http://scielo.iec.pa.gov.br/scielo.php?pid=S167949742006000400004\&script=sci_arttext.

20. Sonis ST, Fazio RC, Fang L. Princípios e práticas de medicina oral. 2a ed. Rio de Janeiro: Guanabara Koogan; 1996.

21. Van Strydonck DA, Slot DE, Van der Velden $U$, Van der Weijden F. Effect of a chlorhexidine mouthrinse on plaque, gingival inflammation and staining in gingivitis patients: a systematic review. J Clin Periodontol [Internet]. 2012 [acesso em: 30 jun 2014];39(11):1042-55. Disponível em: http://dx.doi.org/10.1111/j.1600-051X.2012.01883.x. 22. Pinto VG. Saúde bucal coletiva. $4^{a}$ ed. São Paulo: Santos; 2008.

23. Ditterich RG, Portero PP, Wambier DS, Pilatti GL, Santos FA. Hábitos de higiene bucal e o papel da motivação no controle mecânico do biofilme dental. Odontol. clín.-cient. 2007;6(2):1238.

24. Pilatti GL, Santos FA. Procedimentos clínicos periodontais relacionados à odontologia restauradora. In: Gomes JC. Estética em clínica odontológica. Curitiba: Maio, 2004. p. 241-68.

25. Goldstein RB, Dugan E, Trachtenberg F, Peli E. The impact of a video intervention on the use of low vision assistive devices. Optom Vis Sci [Internet]. 2007 [acesso em: 30 jun

2014];84(3):208-17. Disponível em:

http://dx.doi.org/10.1097\%2FOPX.0b013e3180339a03.

Artigo recebido em 25/03/2013.

Aprovado para publicação em 21/03/2014.

Artigo publicado em 30/06/2014. 